\title{
Software Fault Prediction Using Data Reduction Techniques
}

\author{
Jagruti R. Patil ${ }^{1}$, Swapnaja Suryawanshi ${ }^{2}$ \\ ${ }^{1}$ Savitribai Phule Pune University, Indira College of Engineering and Management, Pune, Maharashtra, India \\ ${ }^{2}$ Professor, Savitribai Phule Pune University, Indira College of Engineering and Management, Pune, Maharashtra, India
}

\begin{abstract}
The procedure of altering bug will be bug triage, which plans to effectively relegate an engineer to another bug. Programming organizations spend the greater part of their expense in managing these bugs. To decrease time and cost of bug triaging, we display a programmed way to deal with foresee a designer with significant experience to comprehend the new coming report. In proposed approach we are doing information decrease on bug information set which will lessen the size of the information and also build the nature of the information. We are utilizing example determination and highlight choice at the same time with verifiable bug information. We have included another module here which will depict the status of the bug like whether it doled out to any engineer or not and it is redressed or not. The objective of bug triaging is to allot possibly experienced designers to new-coming bug reports. To diminish time and cost of bug triaging, we introduce a programmed way to deal with anticipate an engineer with important experience to understand the new coming report. In this paper, we examine the utilization of five term determination techniques on the precision of bug task. Moreover, we re-adjust the heap between engineers taking into account their experience. We lead investigates four genuine datasets. The exploratory results demonstrate that by selecting a little number of separating terms, the F-score can be altogether made strides.
\end{abstract}

Keywords: Bug, Bug triage, Data Reduction, Instance selection, Data Mining.

\section{Introduction}

Programming organizations spend more than 45 percent of expense in managing programming bugs. An unavoidable stride of settling bugs is bug triage, which expects to effectively allot a designer to another bug. To diminish the time cost in manual work, content arrangement procedures are connected to direct programmed bug triage. In this paper, we address the issue of information lessening for bug triage, i.e., how to decrease the scale and enhance the nature of bug information. We join occurrence choice with highlight determination to all the while diminish information scale on the bug measurement and the word measurement. To decide the request of applying occasion determination and highlight choice, we concentrate properties from recorded bug information sets and assemble a prescient model for another bug information set. We experimentally explore the execution of information decrease on bug reports of two substantial open source ventures, in particular Eclipse and Mozilla. The outcomes demonstrate that our information lessening can viably diminish the information scale and enhance the precision of bug triage. Our work gives a way to deal with utilizing methods on information handling to frame decreased and astounding bug information in programming improvement and support. A period expending venture of taking care of programming bugs is bug triage, which means to dole out a right designer to settle another bug. In conventional programming advancement, new bugs are physically triaged by a specialist engineer, i.e., a human triage. Because of the substantial number of day by day bugs and the absence of aptitude of the considerable number of bugs, manual bug triage is costly in time cost and low in precision. In manual bug triage in Eclipse, percent of bugs are relegated by oversight while the time expense between opening one bug and its first triaging is 19.3 days by and large. To maintain a strategic distance from the costly cost of manual bug triage, existing work has proposed a programmed bug triage approach, which applies content characterization methods to foresee engineers for bug reports. In this methodology, a bug report is mapped to a record and a related engineer is mapped to the mark of the archive. At that point, bug triage is changed over into an issue of content characterization and is consequently explained with full grown content grouping systems, e.g., Naive Bayes. Taking into account the consequences of content characterization, a human triage allots new bugs by joining his/her mastery. To enhance the exactness of content characterization methods for bug triage, some further procedures are explored, e.g., a hurling diagram approach and a collective sifting methodology. Nonetheless, vast scale and low-quality bug information in bug stores obstruct the procedures of programmed bug triage. .Since programming bug information are a sort of freestyle content information (created by engineers), it is important to produce very much handled bug information to encourage the application $[1][3][5]$.

\subsection{Problem Specification}

There are two problems related to bug data that may affect the effective use of bug repositories in software development tasks, namely the large scale and the low quality. In a bug repository, a bug is maintained as a bug report, which records the textual description of reproducing the bug and updates according to the status of bug fixing. Reducing bug data set in order to have less scale of data and quality data. For that we have used feature selection and instance selection techniques of data mining as well as we have used historical data.

\subsection{Proposed Methodology}

In this paper, we address the issue of information diminishment for bug triage, i.e., how to lessen the bug information to spare the work expense of engineers and 


\section{International Journal of Science and Research (IJSR) \\ ISSN (Online): 2319-7064 \\ Index Copernicus Value (2013): 6.14 | Impact Factor (2014): 5.611}

enhance the quality to encourage the procedure of bug triage. Information diminishment for bug triage expects to manufacture a little scale and top notch set of bug information by evacuating bug reports and words, which are excess or non-educational. In our work, we consolidate existing strategies of case determination and highlight choice to at the same time diminish the bug measurement and the word measurement. The diminished bug information contain less bug reports and less words than the first bug information and give comparable data over the first bug information. We assess the lessened bug information as per two criteria: the size of an information set and the exactness of bug triage. To stay away from the predisposition of a solitary calculation, we exactly inspect the consequences of four occasion determination calculations and four component choice calculation.

\section{Architecture}

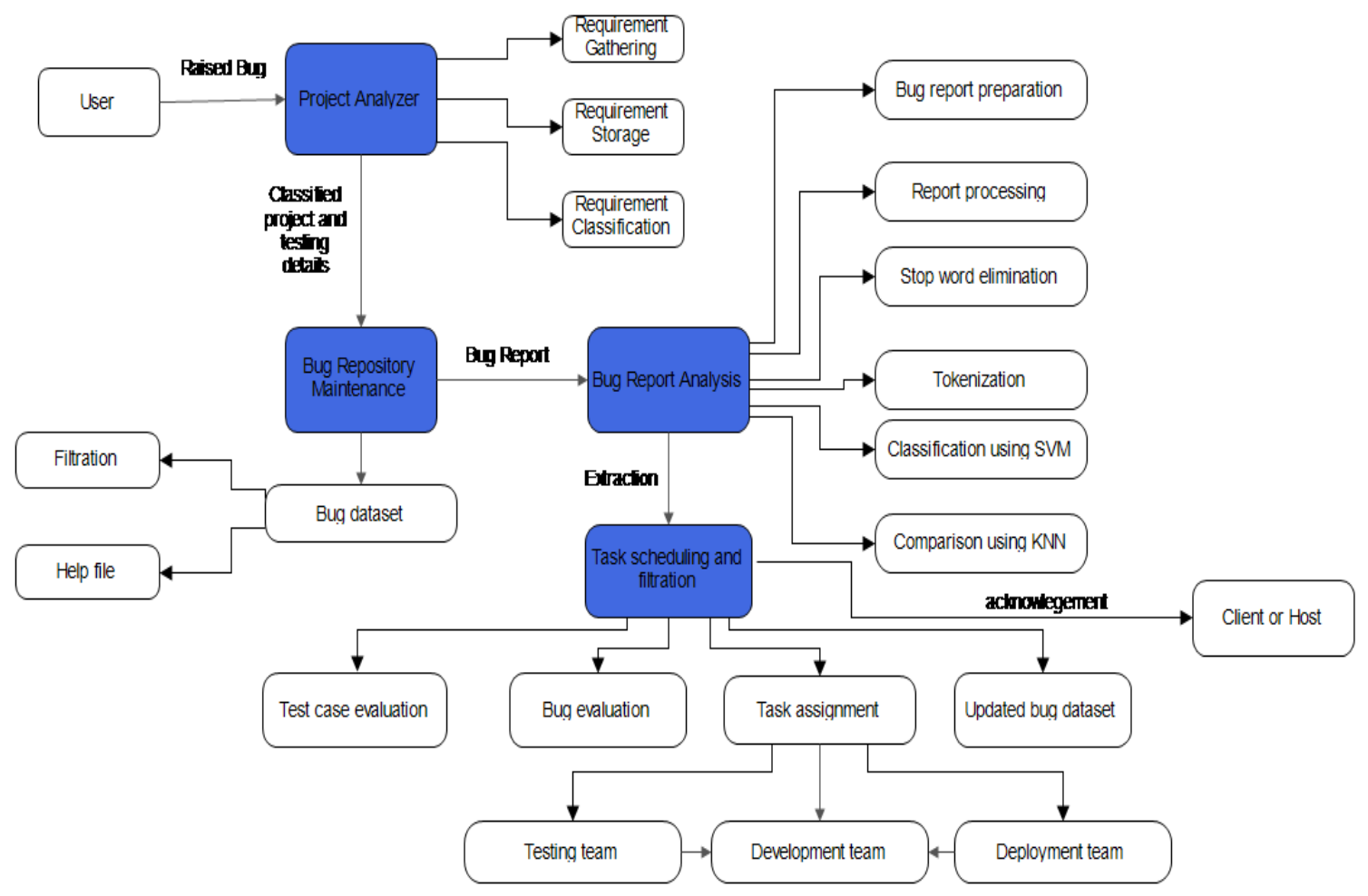

Figure 1: Bug Report Reduction Technique

\subsection{Steps in Model Execution}

1. Project Analyser

2. Bug Repository Maintenance

3. Bug Report Analysis

4. Task Scheduling \& Notification

\section{Project Analyser}

Project Analyser enhances user to maintain all the information regarding project and The process of gathering requirements is usually more than simply asking the users what they need and writing their answers down. Depending on the complexity of the application, the process for gathering requirements has a clearly defined process of its own. This process consists of a Software breakdown structure, module specification, their development strategy, modular dependencies, database, team details, manager, developer, tester, deployment testing team, services details, external reference used for project or module development, third party tools, Module Input Output Details, etc from company. group of repeatable processes that utilize certain techniques to capture, document, communicate, and manage requirements. Module is intended to gather all the information of software development, like
This formal process, which will be developed in more detail, consists of four basic steps.

1. Elicitation - I ask questions, We Communicate.

2. Validation - I analyse, We follow-up questions

3. Specification - I document, we follow-up questions

4. Verification - We all agree.

\section{Bug Repository Maintenance :}

The Bug Repository dataset is a collection of models and metrics of software systems and their histories. The goal of such a dataset is to allow people to compare different bug prediction approaches and to evaluate whether a new technique is an improvement over existing ones. In particular, the dataset contains the data needed to:

1. Run a prediction technique based on source code metrics and/or historical measures and/or process information (cvs logs data);

2. Compute the performance of the prediction by comparing its results with an set, i.e., the number post release defects reported in bug tracking system.

The dataset is designed to perform bug prediction at the class level. However package or subsystem information can be derived by aggregating class data, since per each class it is specified the package that contains it. 


\section{International Journal of Science and Research (IJSR)}

ISSN (Online): 2319-7064

Index Copernicus Value (2013): 6.14 | Impact Factor (2014): 5.611

\section{Bug Report Analysis}

We present an approach for automatic triaging by recommending one experienced developer for each new bug report.

A. Representation Framework:

We have a collection of bug reports, $B=\{b 1, \ldots, b|B|\}$. Each bug report has a collection of term, $\mathrm{T}=\{\mathrm{t} 1, \ldots, \mathrm{t}|\mathrm{T}|\}$, and $\mathrm{a}$ class label (developer), $\mathrm{c} \square$

$\mathrm{C}=\{\mathrm{c} 1, \ldots, \mathrm{c}|\mathrm{C}|\}$.

B. Term selection methods:

Are used to reduce the high dimensionality of term space by selecting the most dis- criminating terms for the classification task. The methods give a weight for each term in which terms with higher weights are assumed to contribute more for the classification task than terms with lower weights.

1. Log Odds Ratio (LOR): Log Odds Ratio measures the odds of the word occurring in the positive class normalized by the negative class. The idea is that the distribution of terms on the relevant documents is different from the distribution of terms on the nonrelevant documents.

2. Chi-Square (X2): In statistics, the $X 2$ test is used to examine independence of two events. The events, $\mathrm{X}$ and $\mathrm{Y}$, are assumed to be independent if $\mathrm{P}(\mathrm{XY})=\mathrm{P}(\mathrm{X}) \mathrm{P}(\mathrm{Y})$. In term selection, the two events are the occurrence of the term and the occurrence of the class.

3. Term Frequency Relevance Frequency (TFRF): The basic behind the TFRF method is that the more high frequency for a term in the positive category than in the negative category, the more contributions it makes in selecting the positive instances from the negative instances.

4. Mutual Information (MI): Mutual information measures the mutual dependence of two random variables. MI computes $\mathrm{X}(\mathrm{t}, \mathrm{c})$ as the mutual information (MI) of term $t$ and class c. MI measures how much the presence and the absence of a term contributes to making the correct classification decision on $\mathrm{c}$.

5. Distinguishing Feature Selector (DFS): DFS is a new novel term selection method. It providesglobal discriminatory powers of the features over the entire text collection rather than being class specific. DFS considers the following requirements: 1) a term that occurs frequently in a single class and not in other classes is distinctive, 2) a term that rarely occurs in a single class and not in other classes is irrelevant, 3 ) a term that occurs frequently in all classes is irrelevant, and 4) a term that occurs in some of the classes is relatively distinctive. DFS assigns scores to the features between 0.5 (least discriminative) and 1.0 (most discriminative).

\section{Task Scheduling \& Notification}

The development group with has run into this issue so many times that we decided to write our own solution to this problem and make solve, this module reaches up to developer as well as customer from Bug Report Analysis phase . It's called Revalue: a signal to arise-and it is a Service that freezes your job requests in overall process until it's time to thaw them out.

\section{Application}

Bug Triage System can be used for any used in any online software or web portals like E-commerce, CRM, CMS, ERP, etc.

\section{Conclusion}

Bug triage is a costly stride of programming upkeep in both work cost and time taken a toll. In this paper, we consolidate feature determination with example choice to lessen the size of bug information sets and in addition enhance the information quality. To deflect mine the request of applying case choice and highlight choice for another bug information set, we concentrate traits of every bug information set and prepare a prescient model taking into account authentic information sets. We experimentally explore the information reduction for bug triage in bug archives of two substantial open source ventures, in particular Eclipse and Mozilla. Our work enhances a way to deal with utilizing methods on information procedure to shape diminished and astounding bug information in programming advancement and support.

\section{References}

[1] Towards graphical models for text processing, Charu C. Aggarwal, Peixiang Zhao

[2] Who Should Fix This Bug?, John Anvik, Lyndon Hiew and Gail C. Murphy

[3] Automatic bug triage using text categorization Davor Cubranic Gail C. Murphy

[4] Another move toward the minimum consistent subset: A tabu search approach to the condensed nearest neighbor rule, Vicente Cerverón Lleó

[5] Efficient Bug Triaging Using Text Mining, Mamdouh Alenezi, Kenneth Magel, Shadi Banitaan

[6] Advances in Instance Selection for Instance-Based Learning Algorithms - HENRY BRIGHTON,CHRIS MELLISH

[7] Information Needs in Bug Reports: Improving Cooperation Between Developers and Users Silvia remraj,Jonathan Sillito, Thomas Zimmermann

[8] The Transformation of Open Source Software

[9] Formal Models for Expert Finding in Enterprise Corporate. 